\title{
Is ultrasound combined with computed tomography useful for distinguishing between primary thyroid lymphoma and Hashimoto's thyroiditis?
}

\author{
Jieli Luo ${ }^{1}$, Fengbo Huang², Panpan Zhou ${ }^{3}$, Jianshe Chen ${ }^{1}$, Yang Sun ${ }^{1}$, Fangting $X u^{1}$, Lilu $W u^{1}$, \\ Pintong Huang ${ }^{1}$ \\ ${ }^{1}$ Department of Ultrasound, The Second Affiliated Hospital of Zhejiang University School of Medicine, Hangzhou, China \\ ${ }^{2}$ Department of Pathology, The Second Affiliated Hospital of Zhejiang University School of Medicine, Hangzhou, China \\ ${ }^{3}$ Department of Radiology, The Second Affiliated Hospital of Zhejiang University School of Medicine, Hangzhou, China
}

\begin{abstract}
Introduction: The aim of the study is to investigate the usefulness of ultrasound combined with computed tomography (CT) for distinguishing between primary thyroid lymphoma (PTL) and Hashimoto's thyroiditis (HT).

Material and methods: The investigation was conducted retrospectively in 80 patients from January 2000 to July 2018. All patients underwent pathological tests to be classified into one of two groups: PTL group and HT group. The cut-off value of CT density was determined using receiver-operating characteristic (ROC) curve analysis. The accuracy, sensitivity, specificity, positive predictive value (PPV), and negative predictive value (NPV) of diagnosis for thyroid by CT alone, ultrasound alone, and the combination of CT plus ultrasound were calculated. Results: Of the 80 study patients, 27 patients were PTL and 53 patients were HT. Mean CT density had a sensitivity of $90.6 \%$ and a specificity of $88.9 \%$ at a cut-off value of $53.5 \mathrm{HU}$, with area under the curve (AUC) 0.88 . Ultrasound combined with CT had the highest specificity, accuracy, and PPV compared with CT alone and ultrasound alone ( $\mathrm{p}$ value $<0.05$ ).

Conclusions: Features such as extremely hypoechogenicity, enhanced posterior echo, cervical lymphadenopathy in ultrasound image, and linear high-density strand signs, and very low density in CT imaging have high sensitivity and specificity in thyroid lymphoma. Therefore, ultrasound combined with CT may be useful for distinguishing between PTL and HT. (Endokrynol Pol 2019; 70 (6): 463-468)

Key words: primary thyroid lymphoma; Hashimoto's thyroiditis; computed tomography density; ultrasound; receiver operating characteristic
\end{abstract}

\section{Introduction}

Primary thyroid lymphoma (PTL) is a rare thyroid tumour, representing approximately $5 \%$ of all thyroid malignancies and accounting for less than $2 \%$ of extranodal malignant lymphomas [1-3]. Despite the rarity of PTL, it is important to recognise PTL promptly because of different management of all the other thyroid neoplasms [4]. PTL occurs mostly in patients aged $60-70$ years, and the incidence in women is higher than in men $[5,6]$. It is estimated that approximately $60-90 \%$ of most PTL cases occur in the background of thyroiditis. One of the many theories that has been proposed to explain the basis is that chronic antigenic stimulation of lymphocytes in autoimmune disorders may lead to malignant transformation [8]. A minority support the theory of aberrant somatic hypermutation [9].

Hashimoto's thyroiditis (HT) is known to be characterised by serological test and ultrasound examination, increased antithyroglobulin and anti- thyroperoxidase antibody, and typical micronodular or microcystic change (1-6 mm) with surrounding echogenic septations in ultrasonic images combined with other clinical information has been considered as suggestive of benign disease [10-12]. By contrast, a few cases of evaluation of PTL by ultrasound and computed tomography (CT) have been reported, with confused and atypical features in the images [13]. The differences in the findings on ultrasound and CT between PTL and HT were not distinctly addressed. In addition, decreased CT density of HT compared with normal thyroid gland has been reported in a small number of cases. However, the difference in CT density between PTL and HT is unclear.

PTL is often curable without extensive surgery if it is diagnosed early and treated appropriately [14, 15]. Therefore, a suspicion of PTL by the radiologist or clinician is important for the early diagnosis and prompt treatment of this potentially curable thyroid malignancy. Therefore, the aim of this study was to investigate the usefulness of ultrasound combined with 
CT for distinguishing between PTL and HT, which can help treatment rapidly.

\section{Material and methods}

\section{Patient selection}

The study was approved by the institutional review board of the institution that provided the images and the Hospital Ethics Committee, and the ethics committee waived the need for written, informed consent from each patient. This investigation was conducted retrospectively among 173 patients using a pathologic thyroid database at our institute between January 2000 and July 2018. Subjects were excluded if they had been treated by chemotherapy and radiotherapy or if they had a history of resection of the thyroid. Certainly, studies lacking outcome measures or comparable results were also excluded. In order to be eligible for inclusion in the study, the data had to meet the following inclusion criteria: PTL or HT that was confirmed by surgical pathology; no previous biopsy before image evaluating or neoadjuvant chemotherapy; detailed examination (ultrasound, CT, serological test, etc.); and clinical information. Finally, 80 patients fulfilled the above criteria and were enrolled in the study. Among them, 27 patients were PTL and 53 patients were HT.

The ultrasound examinations were performed by radiologists using 5-12 MHz linear array transducers. All images were obtained from patients who were scanned in a supine position with neck hyperextension in transverse and longitudinal planes. The three sonographic features were as follows: marked hypoechogenicity was defined as decreased echogenicity compared with neck strap muscle; cervical lymphadenopathy was defined as lymph nodes presenting with measurements of $5 \mathrm{~mm}$ or greater in the short axis, and the absence of a hyperechoic hilum, chaotic vascularity, calcification, a heterogeneous echotexture, or a cystic change; and posterior acoustic enhancement was defined as posterior echogenicity higher than the degree of the lesion.

The CT scan parameters used were as follows: thickness, $4.0 \mathrm{~mm}$; spaces between slices, $4.0 \mathrm{~mm} ; 120.0 \mathrm{kV} ; 165.0 \mathrm{mAs} ; 330.0 \mathrm{~mA}$; field of view, $50 \mathrm{~cm}$; matrix size, $512 \times 512$; and field reconstruction. We measured mean/min/max Hounsfield units of the thyroid on non-contrast CT image as CT number or CT value. In each case, a circular region of interest (ROI) of more than $30 \mathrm{~mm}^{2}$ in diameter was placed on one lobe with homogeneous density, without any calcification and also with minimal partial volume artefact. We measured five sections of the thyroid in order to avoid artefacts or areas of inhomogeneity. All the patients underwent contrast-enhanced scanning, which was attained with a rapid infusion of $100 \mathrm{~mL}$ of $65 \%$ iodinated contrast material administered intravenously. The cut-off value of CT density was obtained by receiver operating characteristic (ROC) curve. Linear high-density strand sign in CT image was defined as linear high density within the lesion.

Two radiologists evaluated the images, with more than eight years of experience. All the radiologists assessed the nodules for three features (marked hypoechogenicity, cervical lymphadenopathy, and posterior acoustic enhancement) in sonograms and one feature (linear high-density strand sign) in CT images. They interpreted the sonograms and CT images independently without any clinical information about the patient, and their consensus was used strictly as the "truth" for the nodule imaging features. These two radiologists met and checked their own selections for agreement after the primary selection. Disagreements were resolved by reaching a consensus through a short discussion and a third investigator was involved if necessary. At least two sonographic features were considered ultrasound positive. CT density < cut-off value or linear high-density strand sign was considered CT positive.

Formalin-fixed tissue was available for haematoxylin-eosin (HE) staining. All of the pathologic specimens were viewed by a pathologist with more than five years' experience in thyroid pathology. In this study, five cases were submitted to the ULCA for consultation because of an undefined result. All the pathology diagnosis was according to the 2018 WHO new classification standard. The PTLs in this study were classified as marginal zone B-cell lymphoma (MALT) or diffuse large B-cell lymphoma (DLBCL) and follicle centre lymphoma (FCL).

\section{Statistical analysis}

The cytology and pathology results served as the reference standard for malignancy. Statistical analyses were performed with SPSS software for Windows v19.0 (IBM, USA). Non-normal distributed data were presented as median (interquartile range). The age and sex distribution of the two groups were determined by the chi-square test and Student's t-test (95\% confidence interval). The cut-off values of CT density were determined using the ROC curve. The level of statistical significance was defined as $p<0.05$ with a confidence interval of $95 \%$.

\section{Results}

Of the 27 PTL patients, nine patients were diagnosed with MALT, 16 patients were diagnosed with DLBCL, and two patients were diagnosed with FCL. Twenty-seven cases of PTL (five males and 22 females), aged 68 years (mean age $68 \pm 14$ years). The tumours involved the right $(n=7)$, left $(n=7)$, or both lobes $(n=5)$ of the thyroid gland. The specific lobe of the thyroid gland involved was unknown in eight cases: 53 cases of HT (12 males and 41 females), aged 63 years (mean age $63 \pm 13$ years). All the patients displayed elevated levels of thyroid antibodies, such as anti-TG or anti-TPO antibodies. The sex distribution of the two groups was not significantly different. The ages in the PTL group were significantly higher than those in patients with HT $(p<0.00)$. Nine patients $(33 \%)$ in the PTL group and 23 patients (43\%) in the HT group presented with a rapidly enlarging anterior neck mass. Other associated symptoms included dyspnoea, hoarseness, dysphagia, and cough in both groups. Six patients in the PTL group and 15 in the HT group had no clinical symptoms. There was no significant difference between two groups in terms of clinical symptoms. Table I summarises the demographic findings in 80 patients.

CT density based on Hounsfield units for the min, max, and average for each lesion was derived from the non-contrast images. The ROC curve used to evaluate the mean CT value, max CT value, and min CT value for differentiation of PTL from HT is shown in Figure 1. As shown in the figure, the area under the ROC curve was 0.89 for mean CT value, 0.78 for max CT value, and 0.68 for min CT value. A statistically significant difference of AUC was observed among mean CT value, max CT value, and min CT value. In our study, the cut-off value of mean CT was 53.5. The sensitivity and specificity of cut-off value were $90.6 \%$ and $88.9 \%$, respectively. The mean CT values in the PTL group were significantly lower than those in patients with HT. Imaging studies of PTL showed the presence of marked hypoechogenicity in 19 patients, posterior acoustic enhancement 
Table I. Comparison of clinical characteristics between patients with primary thyroid lymphoma (PTL) and Hashimoto's thyroiditis (HT)

\begin{tabular}{lccc}
\hline Clinical findings & PTL $(\mathbf{n}=\mathbf{2 7})$ & HT $(\mathbf{n}=\mathbf{5 3})$ & $\mathbf{p}$-value \\
\hline Gender & & & 0.67 \\
\hline Women & 22 & 41 & \\
\hline Men & 5 & 12 & \\
\hline Age (years) & & & $0.00^{*}$ \\
\hline Range & $37-91$ & $54-87$ & \\
\hline Median & 68 & 63 & \\
\hline Interquartile range & 14 & 13 & \\
\hline Symptoms & & & \\
\hline Dyspnoea & 4 & 2 & \\
\hline Hoarseness & 2 & 0 & \\
\hline Dysphagia & 5 & 10 \\
\hline Cough & 1 & 3 \\
\hline Neck enlargement & 9 & 23 \\
\hline No symptom & 6 & 15 \\
\hline * $<0.05$, difference was statistically significant
\end{tabular}

in 17 patients, cervical lymphadenopathy in 16 patients, linear high-density strand sign in 17 patients, and CT density $<53.5 \mathrm{HU}$ in 24 patients. For HT imaging studies, 24 of our patients were marked hypoechogenicity (45\%), 20 were posterior acoustic enhancement (38\%), 28 were cervical lymphadenopathy (53\%), 18 were linear high-density strand sign (34\%), and five were CT density $<53.5(9 \%)$. The specific data are shown in Table II.

The sensitivity, specificity, accuracy, positive predictive value (PPV), and negative predictive value (NPV) of single ultrasound were $70.4 \%, 64.2 \%, 66.3 \%, 50.0 \%$, and $81.0 \%$, respectively, and for CT they were were $85.2 \%, 58.5 \%, 67.5 \%, 51.1 \%$, and $88.6 \%$, respectively. After ultrasound and CT data were combined, the sensitivity, specificity, accuracy, PPV, and NPV were $63.0 \%, 96.2 \%, 85.0 \%, 89.5 \%$, and $83.6 \%$, respectively. For the combined data compared with either ultrasound alone or CT alone, the specificity, accuracy, and PPV were statistically significant $(p=0.000,0.001 ; p=0.006$, 0.009 ; and $\mathrm{p}=0.004,0.004$, respectively). Table III lists the sensitivity, specificity, PPV, NPV, and diagnostic

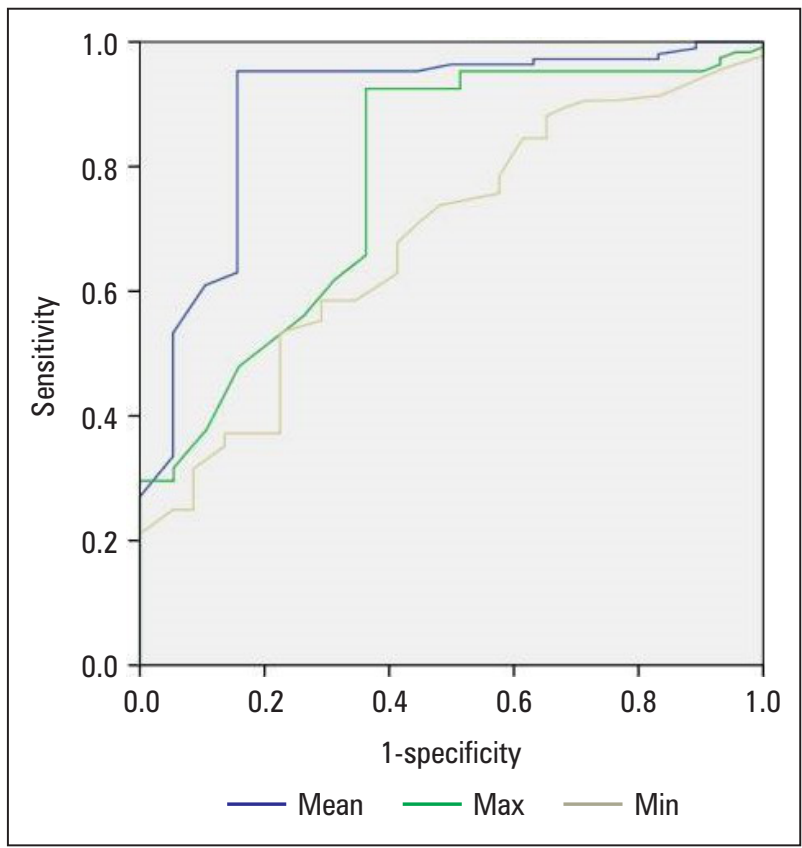

Figure 1. Receiver-operating characteristic (ROC) curve that evaluated the mean computed tomography (CT) number, max CT number, min CT number for differentiating primary thyroid lymphoma (PTL) from Hashimoto's thyroiditis (HT)

accuracy for ultrasound, CT, and combined ultrasound and CT in detail.

There was showing a case as follows. A 65-year-old woman visited an endocrinology department complaining of sudden neck enlargement for less than three months. The lesion was not painful. The results of all laboratory studies, including complete blood count, liver function tests, and thyroid hormone, were unremarkable, although anti-TG and anti-TPO antibodies extremely elevated. Her medical and family histories were remarkable. Ultrasonography revealed posterior acoustic enhancement and cervical lymphadenopathy (Fig. 2). Enhanced CT scans of the neck showed linear high-density sign and CT mean density 34 HU. A biopsy specimen was obtained from the lesion. The histological study revealed DLBCL. Hematoxylin and eosin (H\&E) staining showed that the tumour was composed of large cells with multiple morphology, abundant cytoplasm, irregular nuclear contour, deep nuclear stain,

Table II. Comparison between features of primary thyroid lymphoma (PTL) and Hashimoto's thyroiditis (HT)

\begin{tabular}{|c|c|c|c|c|c|}
\hline & \multicolumn{3}{|c|}{ Ultrasound feature } & \multicolumn{2}{|c|}{ CT feature } \\
\hline & $\begin{array}{c}\text { Marked } \\
\text { hypoechoic }\end{array}$ & $\begin{array}{c}\text { Cervical } \\
\text { lymphadenopathy }\end{array}$ & $\begin{array}{c}\text { Posterior acoustic } \\
\text { enhancement }\end{array}$ & $\begin{array}{l}\text { Linear echogenic } \\
\text { strand signs }\end{array}$ & $\begin{array}{l}\text { CT density } \\
<53.5 \mathrm{HU}\end{array}$ \\
\hline PTL (27) & $19(70.4 \%)$ & $16(59.3 \%)$ & $17(63.0 \%)$ & $17(63.0 \%)$ & $24(88.9 \%)$ \\
\hline HT (53) & 24 (45.3\%) & $28(52.8 \%)$ & $20(37.7 \%)$ & $18(34.0 \%)$ & $5(9.4 \%)$ \\
\hline
\end{tabular}

CT — computed tomography; HU — Hounsfield units 
Table III. Diagnostic performance of ultrasound, computed tomography (CT), and combined ultrasound and CT

\begin{tabular}{|c|c|c|c|c|c|c|c|c|}
\hline \multirow{2}{*}{$\begin{array}{l}\text { Examination } \\
\text { method }\end{array}$} & & \multicolumn{2}{|c|}{ Pathology } & \multirow{2}{*}{ Accuracy } & \multirow{2}{*}{ Sensitivity } & \multirow{2}{*}{ Specificity } & \multirow{2}{*}{ NPV } & \multirow{2}{*}{ PPV } \\
\hline & & Malignant & Benign & & & & & \\
\hline \multirow{2}{*}{ Ultrasound } & Malignant & 19 & 19 & \multirow{2}{*}{$66.3 \%$} & \multirow{2}{*}{$70.4 \%$} & \multirow{2}{*}{$64.2 \%$} & \multirow{2}{*}{$81.0 \%$} & \multirow{2}{*}{$50.0 \%$} \\
\hline & Benign & 8 & 34 & & & & & \\
\hline \multirow{2}{*}{ CT } & Malignant & 23 & 22 & \multirow{2}{*}{$67.5 \%$} & \multirow{2}{*}{$85.2 \%$} & \multirow{2}{*}{$58.5 \%$} & \multirow{2}{*}{$88.6 \%$} & \multirow{2}{*}{$51.1 \%$} \\
\hline & Benign & 4 & 31 & & & & & \\
\hline \multirow{2}{*}{$\begin{array}{l}\text { Combined } \\
\text { ultrasound and CT }\end{array}$} & Malignant & 17 & 2 & \multirow{2}{*}{$85.0 \%$} & \multirow{2}{*}{$63.0 \%$} & \multirow{2}{*}{$96.2 \%$} & \multirow{2}{*}{$83.6 \%$} & \multirow{2}{*}{$89.5 \%$} \\
\hline & Benign & 10 & 51 & & & & & \\
\hline
\end{tabular}

CT — computed tomography; NPV — negative predictive value; PPV — positive predictive value

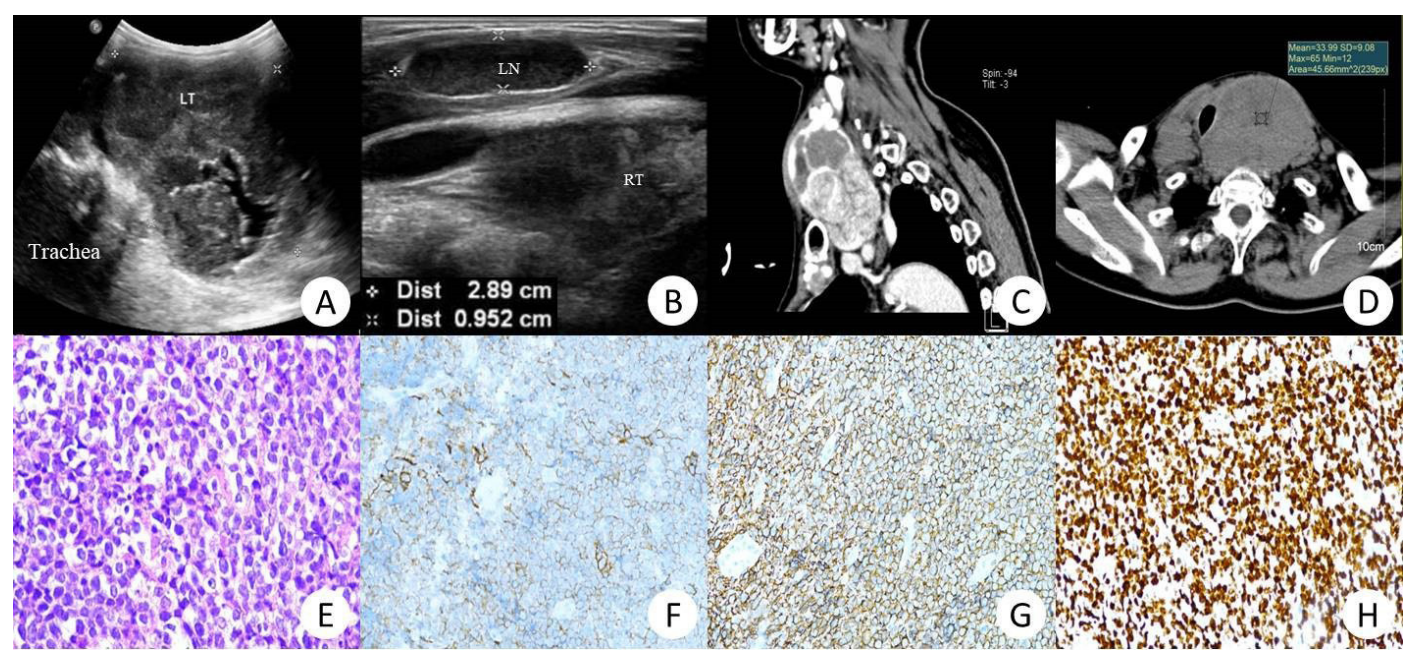

Figure 2. A 65-year-old woman with diffuse large B-cell lymphoma (DLBCL). Longitudinal sonogram of left lobe shows posterior acoustic enhancement $(\boldsymbol{A})$ and cervical lymphadenopathy $(\boldsymbol{B})$. Longitudinal enhanced computed tomography $(\mathrm{CT})$ scan shows presence of linear high-density sign (C). The transverse CT scan shows mean density of tumour $34 \mathrm{HU}(\boldsymbol{D})$. DLBCL lymphoma of thyroid highlighting the abnormal lymphocyte in hematoxylin and eosin (HEE) stain (E). Immunohistochemical stain shows positive CD10 (F) and CD 20 (G). Nuclear proliferation index as assessed by Ki-67 staining was approximately $90 \%$ (F)

and nucleolus. Immunohistochemical studies showed uniform expression of CD20, CD79a, CD10, Bcl-6, MUM-1, and CD21 and absence of Bcl-2, c-Myc, CD5, CD23, and cyclin-D1. The nuclear proliferation index as assessed by Ki-67 staining was approximately $90 \%$. The patient was treated with eight courses of a standard CHOP (cyclophosphamide, doxorubicin, vincristine, and prednisone) chemotherapy regimen. She showed an excellent response to chemotherapy presenting shrinkage of thyroid volume.

\section{Discussion}

The five-year survival rate of thyroid lymphoma with stage IE disease is as high as 75-89\%, while year-year survival rate with stage IIE disease falls to $25-40 \%$ [16]. Therefore, the prognosis for thyroid lymphoma depends on staging, and early diagnosis is of vital importance [17-21]. When patients have coexistent HT with a rapidly growing thyroid mass or with symptoms of obstruction, a likely diagnosis may be HT with or without malignant lymphoma [22]. Ultrasound is a widely accepted diagnostic method for examining thyroid [23-27], but several studies have reported that it is less useful in differentiating between PTL and HT [28]. Ultrasound suffers the limitations of being non-specific [29-31]. CT has been introduced to an augment technique of diagnosis of thyroid disease [32]. However, if CT demonstrates low-attenuation mass in thyroid with additional features, for example, linear high-density strand sign, plus ultrasound evidence of marked hypoechogenicity, enhanced posterior echo, or cervical lymphadenopathy, the diagnosis is probably thyroid lymphoma [33].

Histologically, the thyroid is composed of follicles [34]. The follicles are filled with colloid that contains thyroid hormones produced from thyroglobulin and iodide, and the volume of colloid was found to be 
three times larger than the volume of the epithelial cells, which suggests that the CT density is high in the normal thyroid as a result of the large amount of colloid present [35]. On the other hand, reduced density not only reflects the decrease of iodine concentration in the thyroid follicles but also represents a decrease in colloid content and an increase in follicular cells [36]. HT induces lymphocyte infiltration into the thyroid, which replace normal follicles and reduces the formation and reserve of thyroid hormones [37]. PTL with sever densely proliferated cells compared with HT, which reduces CT density significantly. Linear high-density strand sign presented a compressed rim of unaffected thyroid tissue displaced in the lesion.

Some sonographic findings of PTL were correlated with pathological findings in certain [38]. Orita et al. indicated that lymphoepithelial lesions containing neoplastic cells were consistent with hypoechoic areas of PTL [39]. Marked hypoechogenicity is the most typical appearance of PTL. Due to the extremely low level of internal echoes, nodular PTLs have been described as "pseudocysts" in previous studies. Fibrotic tissue and normal thyroid tissue were consistent with echogenic structures within or around the lesions. Densely and uniformly proliferated lymphoma cells in the lesion were possibly related with an enhanced posterior echo [40]. HT is considered as a risk factor for thyroid lymphoma development. In our study, all patients had a history of HT. Lymphoma was a malignant tumour originating from the lymphatic haematopoietic system leading to the enlargement of cervical lymph nodes [41].

Our results should be interpreted with an understanding of our study limitations. It is not certain that ultrasound combined with $\mathrm{CT}$ can definitely distinguish the PTL from HT. Also, IgG4 chronic thyroiditis will have these three characteristics (marked hypoechogenicity, cervical lymphadenopathy, posterior acoustic enhancement). These patients will present as a false positive in their evaluation, resulting in a reduced PPV. Our study suggested that ultrasound combined with CT could be a diagnostic approach to diagnosis of PTL and HT; however, as we expected, there are some potential biases and limitations in our research. One of the major limiting factors is the selection bias. Also, the simple aspect of our study indicates that further well-designed and prospective studies are needed to verify our results.

\section{Conclusions}

This study evaluated the clinical value of ultrasound and CT features in differentiating between PTL and HT. Neither CT- nor ultrasound-specific features seem to have great diagnostic potential to differentiate between
PTL and HT. Thus, we support the recommendation that ultrasound combined with CT could be used in clinical diagnosis as a noninvasive diagnostic approach, which may lead to a great improvement in accurate and early diagnosis, and for PTL, early preoperative diagnosis is crucial to help make the treatment protocol and improve the patient's prognosis.

\section{Funding information}

National Natural Science Foundation of China, grant numbers 81420108018, 81527803, and 81671707. China ultrasound physician technology star program, Grants number KJXX2018003.

\section{Authorship}

P.H. designed this study. J.L., F.H., P.Z., J.C., Y.S., F.X., L.W. acquired the data. P.H. interpreted the data. J.L. wrote the main manuscript text. All authors reviewed the manuscript. The authors wish to thank H.Z. for discussions about the study design. The authors confirm no conflict of interests related to this study.

\section{References}

1. Perez-Cornago A, Appleby PN, Tipper S, et al. Prediagnostic circulating concentrations of plasma insulin-like growth factor-I and risk of lymphoma in the European Prospective Investigation into Cancer and Nutrition. Int J Cancer. 2017; 140(5): 1111-1118, doi: 10.1002/ijc.30528, indexed in Pubmed: 27870006

2. Borrelli N, Denaro M, Ugolini C, et al. miRNA expression profiling of ,noninvasive follicular thyroid neoplasms with papillary-like nuclear features' compared with adenomas and infiltrative follicular variants of papillary thyroid carcinomas. Mod Pathol. 2017; 30(1): 39-51, doi: 10.1038/modpathol.2016.157, indexed in Pubmed: 27586203.

3. Falduto A, Cimino F, Speciale A, et al. How gene polymorphisms can influence clinical response and toxicity following R-CHOP therapy in patients with diffuse large B cell lymphoma. Blood Rev. 2017; 31(4): 235-249, doi: 10.1016/j.blre.2017.02.005, indexed in Pubmed: 28262268.

4. Karmali R, Nabhan C, Petrich AM, et al. Impact of treatment variability on survival in immuno-competent and immuno-compromised patients with primary central nervous lymphoma. Br J Haematol. 2017; 177(1): 72-79, doi: 10.1111/bjh.14522, indexed in Pubmed: 28211579.

5. Hude I, Sasse S, Engert A, et al. The emerging role of immune checkpoint inhibition in malignant lymphoma. Haematologica. 2017; 102(1): 30-42, doi: 10.3324/haematol.2016.150656, indexed in Pubmed: 27884973.

6. Vater I, Montesinos-Rongen M, Schlesner M, et al. The mutational pattern of primary lymphoma of the central nervous system determined by whole-exome sequencing. Leukemia. 2015; 29(3): 677-685, doi: 10.1038/leu.2014.264, indexed in Pubmed: 25189415.

7. Adams HJA, Kwee TC. Critical considerations on the predictive value of end-of-treatment FDG-PET in lymphoma. Eur J Nucl Med Mol Imaging. 2017; 44(2): 342-343, doi: 10.1007/s00259-016-3553-8, indexed in Pubmed: 27783106.

8. Carbone A, Gloghini A, Caruso A, et al. The impact of EBV and HIV infection on the microenvironmental niche underlying Hodgkin lymphoma pathogenesis. Int J Cancer. 2017; 140(6): 1233-1245, doi: 10.1002/ijc.30473, indexed in Pubmed: 27750386.

9. Burns A, Alsolami R, Becq J, et al. Whole-genome sequencing of chronic lymphocytic leukaemia reveals distinct differences in the mutational landscape between IgHV and IgHV subgroups. Leukemia. 2018; 32(2): 332-342, doi: 10.1038/leu.2017.177, indexed in Pubmed: 28584254.

10. Zaghlol RY, Haghighi A, Alkhayyat MM, et al. Consanguinity and the Risk of Hashimoto's Thyroiditis. Thyroid. 2017; 27(3): 390-395, doi: 10.1089/thy.2016.0495, indexed in Pubmed: 28061551.

11. Hu S, Rayman MP. Multiple Nutritional Factors and the Risk of Hashimoto's Thyroiditis. Thyroid. 2017; 27(5): 597-610, doi: 10.1089/thy.2016.0635, indexed in Pubmed: 28290237.

12. Perga S, Martire S, Montarolo F, et al. The Footprints of Poly-Autoimmunity: Evidence for Common Biological Factors Involved in Multiple Sclerosis and Hashimoto's Thyroiditis. Front Immunol. 2018; 9: 311, doi: 10.3389/fimmu.2018.00311, indexed in Pubmed: 29527211. 
13. Barrantes-Freer A, Engel AS, Rodriguez-Villagra OA, et al. Diagnostic red flags: steroid-treated malignant CNS lymphoma mimicking autoimmune inflammatory demyelination. Brain Pathol. 2018; 28(2): 225-233, doi: 10.1111/bpa.12496, indexed in Pubmed: 28213912.

14. Huang Yu, Jia Bo, Jiang S, et al. Different clinical characteristics and treatment strategies for patients with localized sinonasal diffuse large B cell lymphoma and extranodal NK/T cell lymphoma. J Hematol Oncol. 2017; 10(1): 7, doi: 10.1186/s13045-016-0368-9, indexed in Pubmed: 28057019.

15. Mihara K, Yoshida T, Takei Y, et al. T cells bearing anti-CD19 and/or anti-CD38 chimeric antigen receptors effectively abrogate primary double-hit lymphoma cells. J Hematol Oncol. 2017; 10(1): 116, doi: 10.1186/s13045-017-0488-x, indexed in Pubmed: 28595585.

16. England CG, Rui L, Cai W. Lymphoma: current status of clinical and preclinical imaging with radiolabeled antibodies. Eur J Nucl Med Mol Imaging. 2017; 44(3): 517-532, doi: 10.1007/s00259-016-3560-9, indexed in Pubmed: 27844106.

17. Chang Yu, Cui M, Fu X, et al. MiRNA-155 regulates lymphangiogenesis in natural killer/T-cell lymphoma by targeting BRG1. Cancer Biol Ther. 2019; 20(1): 31-41, doi: 10.1080/15384047.2018.1504721, indexed in Pubmed: 30299211.

18. Lubitz CC, De Gregorio L, Fingeret AL, et al. Measurement and Variation in Estimation of Quality of Life Effects of Patients Undergoing Treatment for Papillary Thyroid Carcinoma. Thyroid. 2017; 27(2): 197-206, doi: 10.1089/thy.2016.0260, indexed in Pubmed: 27824301.

19. Mathur R, Sehgal L, Havranek O, et al. Inhibition of demethylase KDM6B sensitizes diffuse large B-cell lymphoma to chemotherapeutic drugs. Haematologica. 2017; 102(2): 373-380, doi: 10.3324/haematol.2016.144964, indexed in Pubmed: 27742770.

20. Redaelli S, Ceccon M, Antolini L, et al. Synergistic activity of ALK and mTOR inhibitors for the treatment of NPM-ALK positive lymphoma. Oncotarget. 2016; 7(45): 72886-72897, doi: 10.18632/oncotarget.12128, indexed in Pubmed: 27662658.

21. Schuhmacher B, Bein J, Rausch T, et al. et al.. JUNB, DUSP2, SGK1, SOCS1 and CREBBP are frequently mutated in T-cell/histiocyte rich large B-cell lymphoma. Haematologica. 2019; 104(2): 330-337, doi: 10.3324/haematol.2018.203224, indexed in Pubmed: 30213827.

22. Marotta V, Sciammarella C, Chiofalo MG, et al. Hashimoto's thyroiditis predicts outcome in intrathyroidal papillary thyroid cancer. Endocr Relat Cancer. 2017; 24(9): 485-493, doi: 10.1530/ERC-17-0085, indexed in Pubmed: 28696209.

23. Arcolia V, Journe F, Wattier A, et al. Galectin-1 is a diagnostic marker involved in thyroid cancer progression. Int J Oncol. 2017; 51(3): 760-770, doi: 10.3892/ijo.2017.4065, indexed in Pubmed: 28677745.

24. Ha EJu, Na DG, Baek JH, et al. US Fine-Needle Aspiration Biopsy for Thyroid Malignancy: Diagnostic Performance of Seven Society Guidelines Applied to 2000 Thyroid Nodules. Radiology. 2018; 287(3): 893-900, doi: 10.1148/radiol.2018171074, indexed in Pubmed: 29465333.

25. Haymart MR, Banerjee M, Reyes-Gastelum D, et al. Thyroid Ultrasound and the Increase in Diagnosis of Low-Risk Thyroid Cancer. J Clin Endocrinol Metab. 2019; 104(3): 785-792, doi: 10.1210/jc.2018-01933, indexed in Pubmed: 30329071.

26. Hoang JK, Middleton WD, Farjat AE, et al. Reduction in Thyroid Nodule Biopsies and Improved Accuracy with American College of Radiology Thyroid Imaging Reporting and Data System. Radiology. 2018; 287(1): 185-193, doi: 10.1148/radiol.2018172572, indexed in Pubmed: 29498593.

27. Kim M, Kim WG, Oh HS, et al. Comparison of the Seventh and Eighth Editions of the American Joint Committee on Cancer/Union for International Cancer Control Tumor-Node-Metastasis Staging System for Differentiated Thyroid Cancer. Thyroid. 2017; 27(9): 1149-1155, doi: 10.1089/thy.2017.0050, indexed in Pubmed: 28635571.
28. Sharma A, Jasim S, Reading CC, et al. Clinical Presentation and Diagnostic Challenges of Thyroid Lymphoma: A Cohort Study. Thyroid. 2016; 26(8): 1061-1067, doi: 10.1089/thy.2016.0095, indexed in Pubmed: 27256107.

29. Clement SC, Kremer LCM, Verburg FA, et al. Balancing the benefits and harms of thyroid cancer surveillance in survivors of Childhood, adolescent and young adult cancer: Recommendations from the international Late Effects of Childhood Cancer Guideline Harmonization Group in collaboration with the PanCareSurFup Consortium. Cancer Treat Rev. 2018; 63: 28-39, doi: 10.1016/j.ctrv.2017.11.005, indexed in Pubmed: 29202445

30. Lauria Pantano A, Maddaloni E, Briganti SI, et al. Differences between ATA, AACE/ACE/AME and ACR TI-RADS ultrasound classifications performance in identifying cytological high-risk thyroid nodules. Eur J Endocrinol. 2018; 178(6): 595-603, doi: 10.1530/EJE-18-0083, indexed in Pubmed: 29626008.

31. Persichetti A, Di Stasio E, Guglielmi R, et al. Predictive Value of Malignancy of Thyroid Nodule Ultrasound Classification Systems: A Prospective Study. J Clin Endocrinol Metab. 2018; 103(4): 1359-1368, doi: 10.1210/jc.2017-01708, indexed in Pubmed: 29408952.

32. Monteiro R, Han A, Etiwy M, et al. Importance of surgeon-performed ultrasound in the preoperative nodal assessment of patients with potential thyroid malignancy. Surgery. 2018; 163(1): 112-117, doi: 10.1016/j. surg.2017.10.005, indexed in Pubmed: 29128184.

33. Freesmeyer M, Winkens T, Kuehnel C, et al. 99mTc-Pertechnetate-SPECT/US Hybrid Imaging Enhances Diagnostic Certainty Compared With Conventional Thyroid Imaging With Scintigraphy and Ultrasound. Clin Nucl Med. 2018; 43(10): 747-748, doi: 10.1097/RLU.0000000000002241, indexed in Pubmed: 30153145.

34. Bulanova EA, Koudan EV, Degosserie J, et al. Bioprinting of a functional vascularized mouse thyroid gland construct. Biofabrication. 2017; 9(3): 034105, doi: 10.1088/1758-5090/aa7fdd, indexed in Pubmed: 28707625.

35. Yoo J, Ahn HS, Kim SJ, et al. Evaluation of Diagnostic Performance of Screening Thyroid Ultrasonography and Imaging Findings of Screening-Detected Thyroid Cancer. Cancer Res Treat. 2018; 50(1): 11-18, doi: 10.4143/crt.2016.600, indexed in Pubmed: 28231691.

36. Symonds CJ, Seal P, Ghaznavi S, et al. Thyroid nodule ultrasound reports in routine clinical practice provide insufficient information to estimate risk of malignancy. Endocrine. 2018; 61(2): 303-307, doi: 10.1007/s12020-018-1634-0, indexed in Pubmed: 29802606.

37. Sterle HA, Barreiro Arcos ML, Valli E, et al. The thyroid status reprograms $\mathrm{T}$ cell lymphoma growth and modulates immune cell frequencies. J Mol Med (Berl). 2016; 94(4): 417-429, doi: 10.1007/s00109-015-1363-2, indexed in Pubmed: 26564151.

38. Lollert A, Gies C, Laudemann $K$, et al. Ultrasound Evaluation of Thyroid Gland Pathologies After Radiation Therapy and Chemotherapy to Treat Malignancy During Childhood. Int J Radiat Oncol Biol Phys. 2016; 94(1): 139-146, doi: 10.1016/j.ijrobp.2015.09.016, indexed in Pubmed: 26545453.

39. Orita Y, Sato Y, Kimura N, et al. Characteristic ultrasound features of mucosa-associated lymphoid tissue lymphoma of the salivary and thyroid gland. Acta Otolaryngol. 2014; 134(1): 93-99, doi: 10.3109/0001 6489.2013.831994, indexed in Pubmed: 24256049.

40. Liao J, Luan Y, Ren Z, et al. Converting Lymphoma Cells into Potent Antigen-Presenting Cells for Interferon-Induced Tumor Regression. Cancer Immunol Res. 2017; 5(7): 560-570, doi: 10.1158/2326-6066.CIR-16-0221, indexed in Pubmed: 28533311.

41. Gupta NK, Nolan A, Omuro A, et al. Long-term survival in AIDS-related primary central nervous system lymphoma. Neuro Oncol. 2017; 19(1): 99-108, doi: 10.1093/neuonc/now155, indexed in Pubmed: 27576871. 